\title{
A Variability-Aware Energy-Minimization Strategy for Subthreshold Circuits
}

\author{
Junya KAWASHIMA $^{\dagger \mathrm{a})}$, Nonmember, Hiroshi TSUTSUI ${ }^{\dagger \mathrm{b})}$, Hiroyuki $\mathrm{OCHI}^{\dagger \mathrm{c})}$, and Takashi SATO $^{\dagger \mathrm{d})}$, Members $^{2}$
}

\begin{abstract}
SUMMARY We investigate a design strategy for subthreshold circuits focusing on energy-consumption minimization and yield maximization under process variations. The design strategy is based on the following findings related to the operation of low-power CMOS circuits: (1) The minimum operation voltage $\left(V_{\mathrm{DD}_{\text {min }}}\right)$ of a circuit is dominated by flip-flops $(\mathrm{FFs})$, and $V_{\mathrm{DD}_{\text {min }}}$ of an FF can be improved by upsizing a few key transistors, (2) $V_{\mathrm{DD}_{\text {min }}}$ of an FF is stochastically modeled by a log-normal distribution, (3) $V_{\mathrm{DD}_{\min }}$ of a large circuit can be efficiently estimated by using the above model, which eliminates extensive Monte Carlo simulations, and (4) improving $V_{\mathrm{DD}_{\min }}$ may substantially contribute to decreasing energy consumption. The effectiveness of the proposed design strategy has been verified through circuit simulations on various circuits, which clearly show the design tradeoff between voltage scaling and transistor sizing.

key words: subthreshold operation, process variation, minimum operation voltage estimation, energy minimization, yield maximization
\end{abstract}

\section{Introduction}

Having more than one processor on an electronic device has already become commonplace with the advancement of integrated circuit technology. The number of such devices that intelligently support our daily lives is still rapidly increasing. Among others, sensor devices, which gather and process information from our surrounding environment, are attracting greater interest [1]-[3]. Applications of those devices span vast areas - from health care, to environmental monitoring, to animal behavior tracking [4]. Sensor devices are required to achieve the lowest energy consumption to maintain the required data processing within a limited power supply capacity. Energy harvesting, from, e.g., solar light [5] or vibrations [6], may be used to assist a battery, but their energy density and availability are usually strictly limited. Both extremely low energy consumption and low peak power consumption are important for these types of circuits.

Supply voltage scaling is one of the most efficient techniques for reducing energy consumption because dynamic power is scaled quadratically to voltage. Lowering supply voltage, in general, reduces both peak power dissipation and total energy consumption. Subthreshold operation, which utilizes low supply voltage near or below transistors' thresh-

\footnotetext{
Manuscript received March 19, 2012.

Manuscript revised June 18, 2012.

${ }^{\dagger}$ The authors are with the Department of Communications and Computer Engineering, the Graduate School of Informatics, Kyoto University, Kyoto-shi, 606-8501 Japan.

a) E-mail: paper@easter.kuee.kyoto-u.ac.jp

b)E-mail: tsutsui@easter.kuee.kyoto-u.ac.jp

c) E-mail: ochi@kuee.kyoto-u.ac.jp

d)E-mail: takashi@i.kyoto-u.ac.jp

DOI: 10.1587/transfun.E95.A.2242
}

old voltages, is considered as a promising technique that can substantially reduce power dissipation and energy consumption [7].

Subthreshold operation has been widely explored in recent years. However, its design methodology is not fully established because it faces several challenging issues. Minimum operation voltage [8] is one of the most important issues. Each circuit has its own minimum operation voltage $\left(V_{\mathrm{DD}_{\text {min }}}\right)$, below which the circuit does not function as designed. Given that the minimum operation voltage is highly sensitive to process variation, it varies from chip to chip. It is difficult, in particular in the case of large-scale logic circuits, to determine a design-dependent optimal supply voltage so that power consumption is minimized and yield is maximized [9]. Another, but related, issue is leakage energy. As the supply voltage decreases, the delay of each gate increases exponentially to the voltage, which lengthens operation time and thus exponentially increases leakage energy.

The goal of this work is to establish a design strategy for subthreshold circuits that minimizes energy consumption and maximizes yield. This paper presents the following results based on extensive circuit simulations using transistor models and standard cells made using a commercial 65nm CMOS technology.

- Robustness in low voltage operation is dominated mainly by flip-flops (FFs), and the robustness is improved drastically by avoiding the use of small transistors.

- The minimum operation voltage $\left(V_{\mathrm{DD}_{\min }}\right)$ distribution of an FF is closely approximated by log-normal distributions. Therefore, the $V_{\mathrm{DD}_{\min }}$ distribution of a largescale circuit can be quickly and accurately estimated through analytical calculations. This modeling enables us to eliminate Monte Carlo circuit simulations on the large-scale circuit, which is infeasible to do in many cases.

- Energy minimization and yield maximization are achieved simultaneously by transistor sizing of an FF, and by finding an operation voltage that achieves minimum energy consumption under a $V_{\mathrm{DD}_{\min }}$ constraint.

Examples of the proposed energy minimization will be demonstrated through designs of three modules in a JPEG encoder, which are different in terms of the total FF count and the ratio between FF and combinational logic gates. Experimental result shows that the choice of suitable operation 
voltages as well as transistor size in an FF can lead to a $20 \%$ energy savings for one of the three modules, a DCT circuit, as compared with the design using nonoptimized FF.

The remainder of this paper is organized as follows. Section 2 reviews the issues in the low supply voltage circuit design, e.g., leakage energy and $V_{\mathrm{DD}_{\min }}$. In Sect. 3, the failure mode in low-voltage operation is studied through circuit simulations of simple circuits, and a method for $V_{\mathrm{DD}_{\min }}$ estimation is proposed. Section 4 proposes a design strategy for subthreshold circuits, which achieves both energy minimization and yield maximization. Also, in Sect. 5, its effectiveness is demonstrated through an example design of a JPEG encoder. Finally, Sect. 6 draws conclusions.

\section{Design Issues in Low Voltage Operation}

\subsection{Energy Consumption}

Power dissipation of a CMOS circuit is expressed by the following equation [10]:

$$
P=\alpha f C V_{\mathrm{DD}}^{2}+\alpha f Q_{\mathrm{SC}} V_{\mathrm{DD}}+I_{\mathrm{LEAK}} V_{\mathrm{DD}} .
$$

Here, $\alpha$ is the switching factor, $f$ is the clock frequency, $C$ is the load capacitance, $Q_{\mathrm{SC}}$ is the electric charge consumed as a short-circuit current, $I_{\text {LEAK }}$ is the leakage current, and $V_{\mathrm{DD}}$ is the supply voltage. The first term represents dynamic power dissipation owing to charging and discharging of the load capacitance. The second term represents dynamic dissipation by a short-circuit current. The third term represents static dissipation from the leakage current. Here, power dissipation by the short-circuit current, the second term in Eq. (1), is relatively smaller than the others and the duration of the short-circuit current is short. Hence, we will focus on the first and the last terms of the equation in this paper.

From Eq. (1), we observe that the power $P$ is reduced by lowering $\alpha, f, C$, or $V_{\mathrm{DD}}$. Among others, supply voltage scaling is extremely effective because it is related to all the terms in Eq. (1) and the first term will be reduced quadratically. This paper focuses on near- or subthreshold operations where $V_{\mathrm{DD}}$ is set around or lower than the threshold voltages of the transistors. To enable subthreshold operations, however, several issues should be considered.

First, supply voltage scaling reduces the overdrive voltage of a transistor and thus reduces its driving current. As a consequence, gate delay increases and circuit performance degrades. Figure 1 shows oscillation periods of a ring oscillator (RO) composed of 101 inverters as a function of the supply voltage. In the low-voltage region, delay increases exponentially as the supply voltage is lowered.

Second, although the power dissipation from the leakage current, the third term in Eq. (1), becomes small as the supply voltage is lowered, the delay increase is more significant since it has an exponential dependence on the supply voltage. As a result, the leakage energy increases when the supply voltage is scaled to a very low value. These effects result in the existence of a minimum point in the plot of total energy consumption against supply voltage [11], as shown

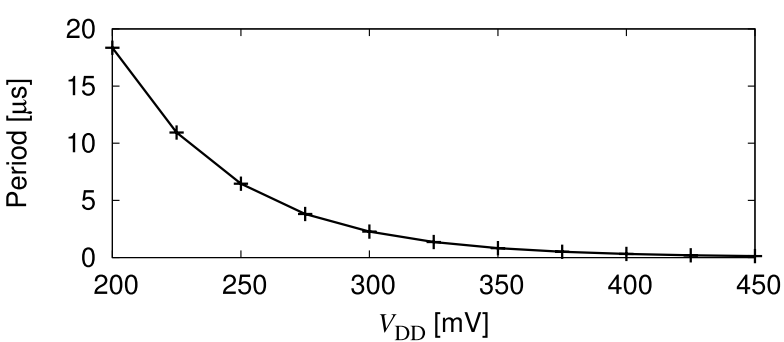

Fig. 1 Oscillation period of a 101-stage inverter-chain ring oscillator as a function of supply voltage $\left(V_{\mathrm{DD}}\right)$.

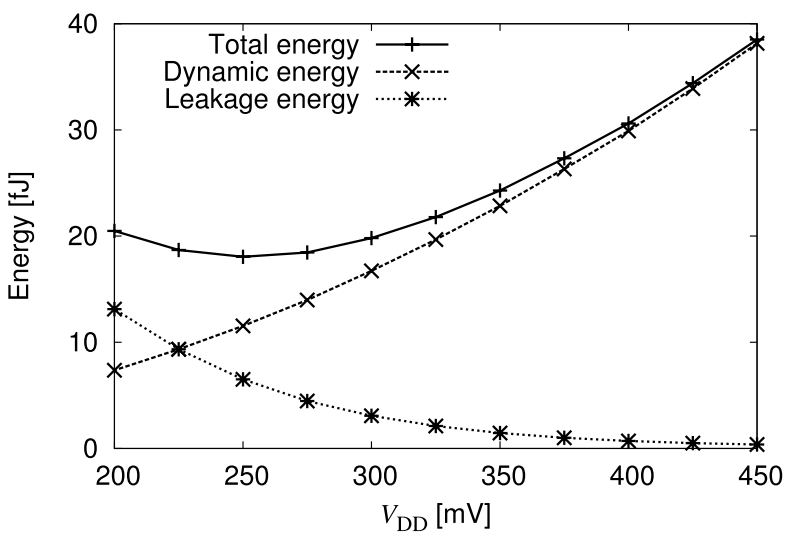

Fig. 2 One-period energy of a 101-stage inverter-chain ring oscillator as a function of supply voltage $\left(V_{\mathrm{DD}}\right)$.

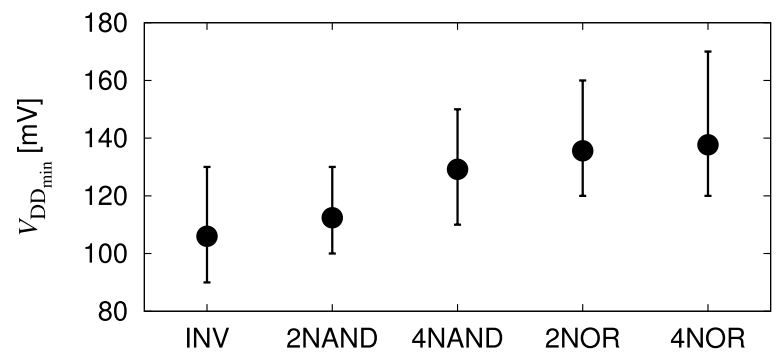

Fig.3 Minimum operation voltages of RO circuits composed of different logic cells.

in Fig. 2. When we pursue energy efficiency, it is desirable for circuits to operate as close to the minimum energy voltage $\left(V_{\mathrm{ENE}_{\min }}\right)$ as possible.

\subsection{Minimum Operation Voltage}

Each circuit has its own minimum operation voltage $\left(V_{\mathrm{DD}_{\min }}\right)$, below which the circuit fails to work correctly. Because of this fact, not all circuits are able to work at its minimum energy voltage $V_{\mathrm{ENE}_{\min }}$.

Figure 3 shows $V_{\mathrm{DD}_{\min }}$ of $5 \mathrm{RO}$ circuits, which are composed of inverter, 2NAND, 4NAND, 2NOR, or 4NOR gates respectively. The number of stages is 101 for all ROs and the results are based on circuit simulations. Error bars show the maximum and the minimum $V_{\mathrm{DD}_{\min }}$ in 100 times circuit simulations applying threshold voltage variation. Figure 3 
indicates that $V_{\mathrm{DD}_{\text {min }}}$ of a combinational circuit is affected by process variation and high fan-in gates tend to have higher $V_{\mathrm{DD}_{\text {min }}}$. Assuming that operating frequency for a $V_{\mathrm{DD}}$ is determined at the design phase, $V_{\mathrm{ENE}_{\min }}$ is relatively insensitive to threshold voltage variation since power consumption is averaged over the chip. On the contrary, $V_{\mathrm{DD}_{\text {min }}}$ is strongly affected by it. It is desirable that $V_{\mathrm{DD}_{\min }}$ is made sufficiently smaller than $V_{\mathrm{ENE}_{\min }}$ by design. This can be achieved by appropriately sizing transistors in standard logic gates.

\section{Yield Modeling at Low-Voltage Operation}

\subsection{Investigation of the Cause of Errors}

A logic circuit fails to work correctly at low voltages for various reasons, such as attenuation of signal amplitude in combinational circuits or write/read failures in FFs. To ensure correct operation of circuits and to improve yield without increasing energy consumption significantly requires clarification of the mechanism of the circuit errors.

To investigate the cause of such errors in low-voltage operation, we first analyze a simple circuit of the target process technology illustrated in Fig. 4. This is a 1-bit counter that consists of an odd number of 4NOR gates (the worst case logic gate in Fig. 3) and an FF. By cascading $k$-4NOR gates, the circuit models a path of a combinational circuit that has a logic depth of $k$. Using this circuit, we can observe behavior of both combinational and sequential circuit components in low supply voltages.

$V_{\mathrm{DD}_{\text {min }}}$ of this circuit is evaluated by circuit simulations. Starting from $1.2 \mathrm{~V}$, the supply voltage is gradually lowered until the FF produces an incorrect output. Transistor models of a commercial 65-nm technology are used. Areadependent threshold voltage variation [12] has been considered in the simulations. To observe the influence of logic depth of combinational circuits, $k$ has been altered from 3 to 31 . In this study, we excluded timing error when $V_{\mathrm{DD}_{\min }}$ is considered, since setup error can be avoided by applying sufficiently low frequency clock and hold error can be avoided by logic synthesis, i.e., removal of short paths.

Figure 5 shows a transistor-level schematic diagram of an FF used in this analysis. For FFs in standard cell libraries that are intended to be used in nominal voltages, it is common for transistors of very small gate width $W$ to be used as feedback and clock inverters. Assuming this to be a major cause of failures in the subthreshold region, we conducted simulations to see how much improvement against process variation is obtained through enlarging the widths of these small transistors.

Figure 6 shows $V_{\mathrm{DD}_{\min }}$ distributions for different logic depths, $k$, and different gate widths, $W$. The distribution of each logic depth is obtained by simulation of 100 times. Figure 6(a) shows the $V_{\mathrm{DD}_{\min }}$ distributions for the standard cell. For this particular combination of process technology and standard cell, $V_{\mathrm{DD}_{\min }}$ is determined by the FF and is almost insensitive to the logic depth of the combinational circuit. Figures 6(b) and (c) show the distributions of $V_{\mathrm{DD}_{\min }}$ for the

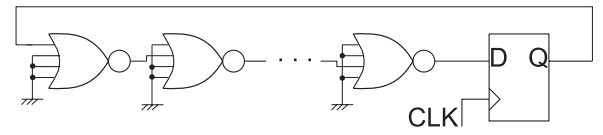

Fig. 4 A circuit composed of an odd number of $k$-4NOR gates and one $\mathrm{FF}$.
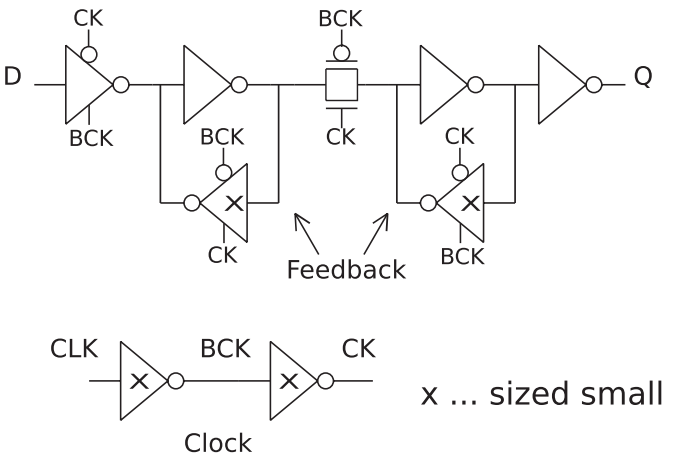

Fig. 5 A transistor-level schematic diagram of an FF. Transistors of very small gate width $W$, which are marked with "x," are used for feedback and clock inverters.

cases where transistor-gate widths are all $1.5 \times$ and $2 \times$, respectively, larger than their original sizes. Here, the transistor sizing is limited only to the small nMOS and pMOS transistors that comprise the logic gates marked " $x$ " in Fig. 5. We can clearly see that the distributions successfully move to lower voltages as we increase transistor sizes. Although these transistors play important roles in an FF (e.g., holding data and distributing clock signal), their default sizes are designed to be small to save area and power. Given that the most influential process variation is threshold voltage variation from random-dopant fluctuation, they are highly sensitive to process variation. It is concluded that $V_{\mathrm{DD}_{\text {min }}}$ will be significantly improved by upsizing small transistors in FFs.

Figure 6 also indicates that the logic depth does not affect $V_{\mathrm{DD}_{\text {min }}}$ very much for different channel widths. The FF can be considered as the dominant factor that determines $V_{\mathrm{DD}_{\min }}$.

\subsection{Efficient Method for Estimating $V_{\mathrm{DD}_{\min }}$}

Considering the above results, we propose a method to estimate $V_{\mathrm{DD}_{\text {min }}}$ for a large circuit. In general, carrying out a circuit simulation on a very large circuit is time consuming. It takes an impractically long time to obtain a statistical distribution of $V_{\mathrm{DD}_{\text {min }}}$ by repeating the simulation to reflect process variations. It is desirable to develop a way to estimate $V_{\mathrm{DD}_{\text {min }}}$ of a large circuit by using simulation results of smaller and simpler circuits.

It is obvious that all FFs in a circuit must work correctly to perform correct operation. Consequently, the probability that the target circuit works correctly at a supply voltage of $V_{\mathrm{DD}}=V_{i}$ is given by the following equation:

$$
P_{V_{i}}=\left(P_{V_{i \mathrm{FF}}}\right)^{N} \text {, }
$$

where $N$ is the number of FFs and $P_{V_{i F F}}$ is the probability 


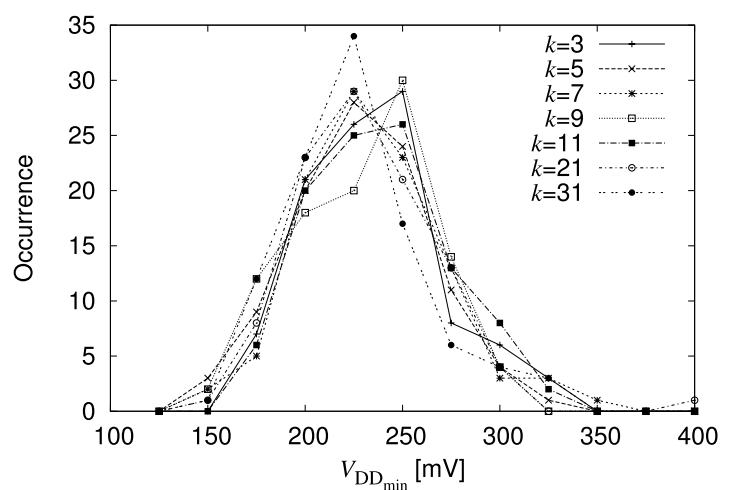

(a) Gate width $W: \times 1$ (standard cell).

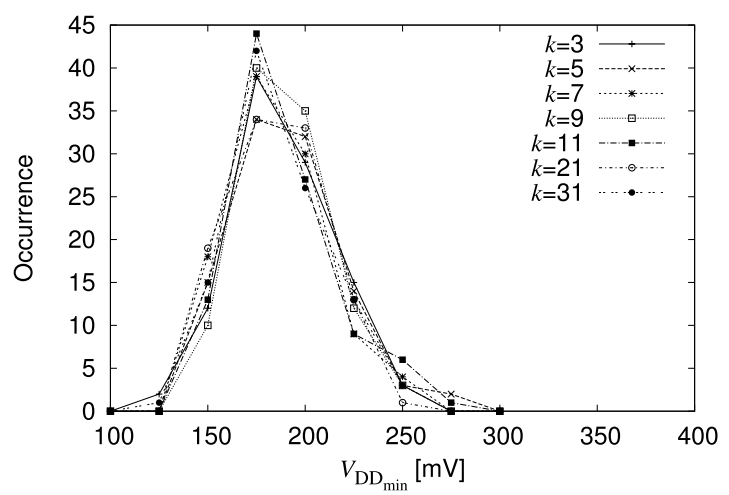

(b) Gate width $W: \times 1.5$.

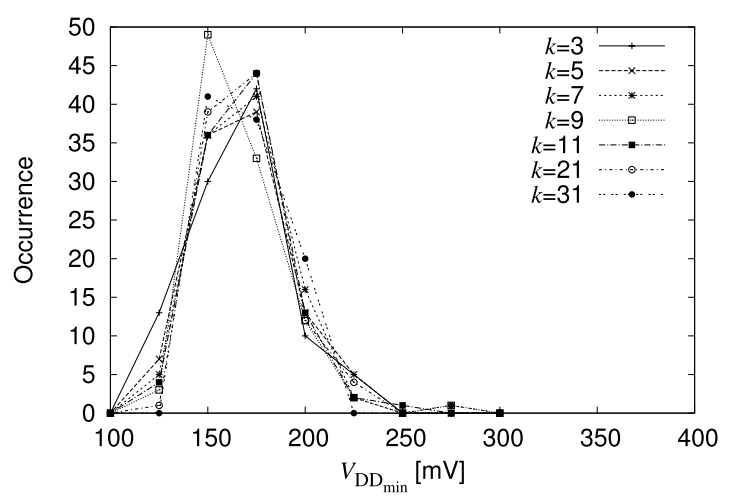

(c) Gate width $W: \times 2$.

Fig. $6 V_{\mathrm{DD}_{\min }}$ distributions of the circuit shown in Fig. 4. The distributions are obtained for different logic depths, $k$, and different gate widths, $W . W: \times 1$ means that the gate widths $W$ of FFs are the same as those of the standard cells.

that the circuit shown in Fig. 4 works correctly at the supply voltage of $V_{i}$. From Eq. (2), even if $P_{V_{i F F}}$ is close to 1, $P_{V_{i}}$ becomes exponentially small when $N$, the number of FFs in a target circuit, is very large. Figure 7 shows the influence of $N$ to $P_{V_{i}}$. When $N=10^{3}$, for example, $P_{V_{i F F}}$ must be accurate to about five decimal places to estimate the $V_{\mathrm{DD}_{\min }}$ that guarantees $95 \%$ yield. This means that we need to know the distribution of $V_{\mathrm{DD}_{\min }}$ very accurately, especially around the high- $V_{\mathrm{DD}}$ region where the lowest bound of the operational voltage is determined. Otherwise, the estimation

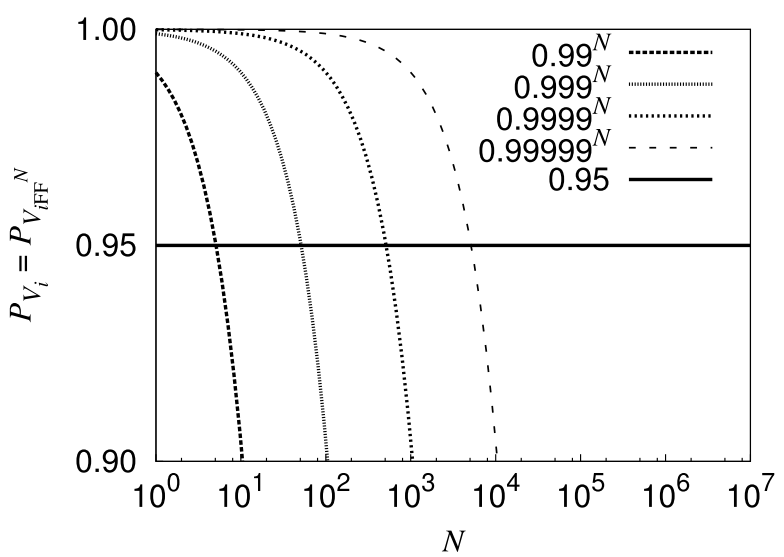

Fig. 7 The probability that the target circuit works correctly at a supply voltage $V_{\mathrm{DD}}=V_{i}$ for different $N$ and $P_{V_{i \mathrm{FF}}} . N$ is the number of FFs in the target circuit and $P_{V_{i F F}}$ is the probability that the circuit shown in Fig. 4 works correctly at the supply voltage of $V_{i}$.

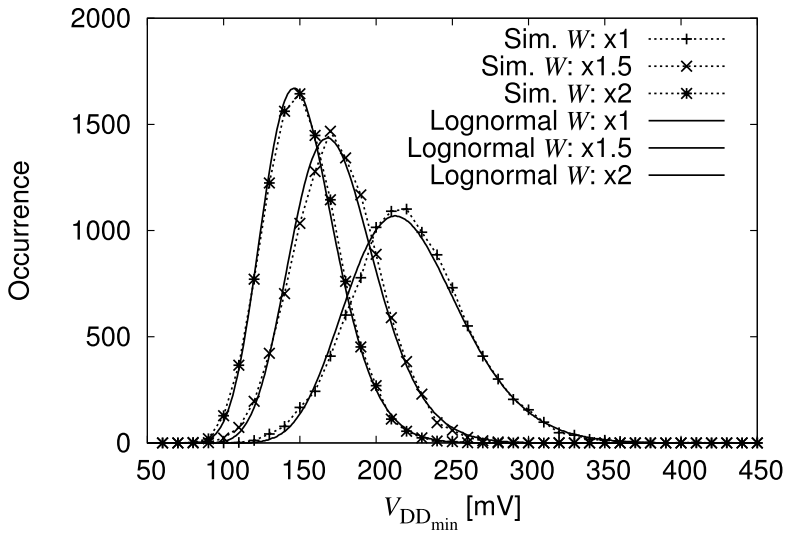

Fig. $8 V_{\mathrm{DD}_{\min }}$ distributions of differently sized FFs. The symbols and dashed lines represent the distributions obtained by SPICE Monte Carlo simulations; the solid lines represent the proposed log-normal distributions modeled from the simulation results.

of the $V_{\mathrm{DD}_{\min }}$ loses confidence and thus a large guard-band would be required for the minimum operation voltage.

Figure 8 shows the distribution of $V_{\mathrm{DD}_{\min }}$ of differently sized FFs. We set the CLK and DATA signals so that both setup and hold times are satisfied. To obtain smoother and reliable histograms we had simulated the circuit 10,000 times. Possibly, tail region needs to be simulated very accurately when $N$ is large. Instead of increasing the number of simulations, we propose modeling the $V_{\mathrm{DD}_{\min }}$ of the circuit by using log-normal distributions from the experimental result of Fig. 8. The approximations using the proposed model are also presented in the same figure. We find very good matches between corresponding simulation results and proposed models.

Using this model, $V_{\mathrm{DD}_{\min }}$ of general circuits will be estimated accurately. Two shape parameters of the log-normal distribution $p(V)$ can be obtained by fitting the histogram of $V_{\mathrm{DD}_{\text {min }}}$ as in Fig. 8 . Then, $P_{V_{i F F}}$ will be given as the following cumulative distribution: 


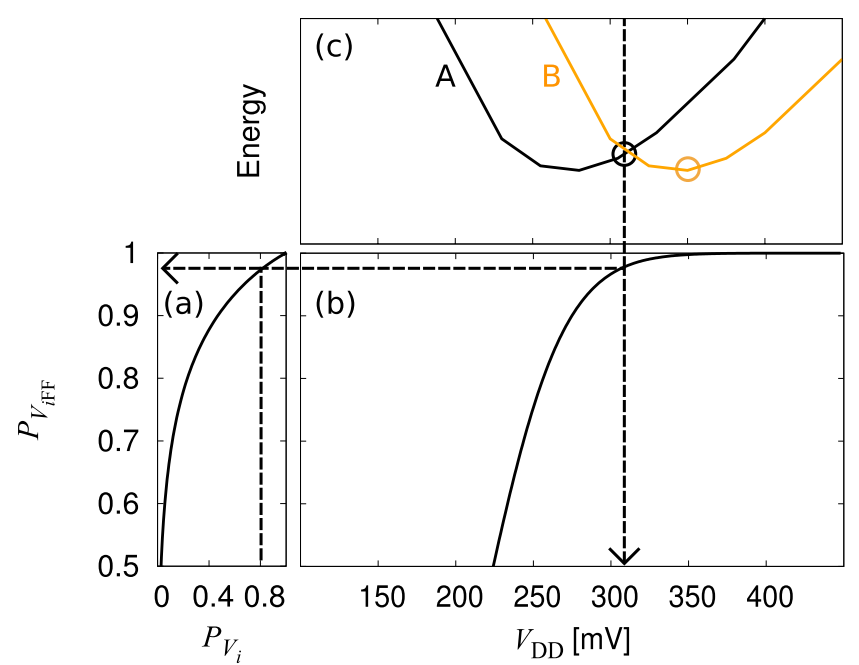

Fig. 9 Illustrative example of the proposed design strategy to find the optimal $V_{\mathrm{DD}}$ for energy minimization under a yield constraint.

$$
P_{V_{i \mathrm{FF}}}=\int_{-\infty}^{V_{i}} p(V) d V .
$$

Substituting Eq. (3) into Eq. (2), we can quickly estimate $V_{\mathrm{DD}_{\min }}$ of a general circuit without executing Monte Carlo simulations.

\section{Design Strategy for Energy Minimization}

In this section, we propose a design strategy for minimum energy consumption by subthreshold circuits while satisfying a given yield constraint. Our strategy consists of the following steps.

Step 1: Estimate the required FF yield $P_{V_{i \mathrm{FF}}}$ to satisfy a circuit yield requirement.

Step 2: Estimate the minimum supply voltage that satisfies the FF yield $P_{V_{i \mathrm{FF}}}$.

Step 3: Obtain the $V_{\mathrm{DD}}$-energy curve

Step 4: Find the optimal $V_{\mathrm{DD}}$ for the subthreshold circuit.

These steps are illustrated in Fig. 9. The vertical axes of Figs. 9(a) and (b) are equal, and, hence, the scale of the vertical axis is omitted for (b). Similarly, the horizontal axes of Figs. 9(b) and (c) are equal, and thus the horizontal axis of (c) is omitted.

\subsection{Step 1: Estimation of $P_{V_{i \mathrm{FF}}}$}

First, we translate the required circuit yield into the yield of an individual FF. The inputs of this step are the required circuit yield $P_{V_{i}}$ and the number of FFs in the circuit, $N$. The output is $P_{V_{i \mathrm{FF}}}$. This translation can be carried out by solving Eq. (2) inversely,

$$
P_{V_{i \mathrm{FF}}}=\sqrt[N]{P_{V_{i}}}
$$

For example, to guarantee an $80 \%$ yield when the target circuit has $100 \mathrm{FFs}$, the required yield for the FF cell is
$P_{V_{i F F}}=\sqrt[100]{0.8}=0.9978$. An example of this translation is graphically presented in Fig. 9(a).

\subsection{Step 2: Estimation of Minimum $V_{\mathrm{DD}}$ to Satisfy FF Yield}

Next, we calculate the minimum $V_{\mathrm{DD}}$ to satisfy the required FF yield obtained in the previous step. The input of this step is $P_{V_{i \mathrm{FF}}}$ and the output is $V_{\mathrm{DD}_{\text {min }}}$. This is carried out according to the following substeps.

1. Derive a log-normal distribution model of FFs, $p(V)$ used in the circuit. The parameters of the log-normal distribution are determined so that the model best approximates the histograms of a Monte Carlo simulation, as shown in Fig. 8. The Monte Carlo simulation is computationally intensive, but it is necessary only when we introduce a new FF cell.

2. Obtain the cumulative distribution $P_{V_{\text {iFF }}}$ by Eq. (3).

3 . Find $V_{\mathrm{DD}_{\text {min }}}$ by reversely looking up the cumulative distribution $P_{V_{i \mathrm{FF}}}$. An example of the look-up is presented as a dotted line in Fig. 9(b).

\subsection{Step 3: Obtaining the $V_{\mathrm{DD}}$ - Energy Curve}

We then obtain the $V_{\mathrm{DD}}$-energy curve as in Fig. 9 (c). This is carried out according to the following two steps.

1. For each supply voltage, find the maximum operating frequency $f_{\max }$ of the circuit. In this paper, we assume that $f_{\max }$ for each supply voltage is determined at the design phase. In other words, we do not assume runtime clock frequency adjustment considering a process variation of the fabricated chip.

Note that the critical path at each supply voltage must be chosen because the critical path may be supply voltage dependent. Calculation of $f_{\max }$ can be conducted, for example, by utilizing static timing analysis (STA) and its library dedicated to low supply voltages. A sufficiently large number of top- $n$ worst paths at the nominal supply voltage may also be used. In our case, 100 candidate paths are extracted using STA at nominal voltage, and then the actual worst-path delay at each supply voltage is calculated by circuit simulations on the candidate paths.

2. For each supply voltage, derive the energy consumption of the circuit at $f_{\max }$. Unlike $V_{\mathrm{DD}_{\min }}$, energy consumption is less sensitive to random process variation because energy variation is averaged over a large number of logic gates in the circuit. This enables us to obtain the energy consumption through only a single run of circuit simulation at the typical process condition.

\subsection{Step 4: Finding the Optimal $V_{\mathrm{DD}}$}

Given the yield constraint, the supply voltage cannot be lower than $V_{\mathrm{DD}_{\min }}$. From the result of Steps 2 and 3, either 
Table 1 The number of FFs and total cell instances of three circuit modules in a JPEG encoder.

\begin{tabular}{c|c|c|c|c}
\hline Module & FFs & $\begin{array}{c}\text { Total cell } \\
\text { instances }\end{array}$ & $\begin{array}{c}\text { FF ratio in } \\
\text { cell count }\end{array}$ & $\begin{array}{c}\text { FF ratio in } \\
\text { area }\end{array}$ \\
\hline \hline VLC & 64 & 2,093 & $3.1 \%$ & $1.9 \%$ \\
\hline DCT & 241 & 1,038 & $23.2 \%$ & $22.3 \%$ \\
\hline Q & 705 & 3,695 & $19.1 \%$ & $21.4 \%$ \\
\hline
\end{tabular}

$V_{\mathrm{ENEmin}}$ or $V_{\mathrm{DD}_{\min }}$ is higher, and thus there are two exclusive conditions.

1. When $V_{\mathrm{ENEmin}}<V_{\mathrm{DD}_{\min }}$ (curve A in Fig. 9(c)): The supply voltage cannot be lowered below $V_{\mathrm{DD}_{\min }}$. Hence the energy-optimized supply voltage becomes $V_{\mathrm{DD}_{\text {min }}}$.

2. When $V_{\mathrm{DD}_{\min }} \leq V_{\mathrm{ENEmin}}$ (curve B in Fig. 9(c)): The supply voltage can be lowered to the energy minimum point $V_{\text {ENEmin }}$, which is the energy-optimized supply voltage.

\section{Experimental Designs of Subthreshold Circuits}

In this section, we apply the proposed design strategy on experimental subthreshold circuits for validation. The circuits used are the following: discrete cosine transform (DCT), quantization $(\mathrm{Q})$, and variable codeword length coding (VLC). These are chosen so that ratios of FFs and combinational counterpart are different because they affect how the minimum energy voltage is determined. Table 1 shows the number of FFs and total number of cell instances in these circuits. These circuits will be optimized by the selections of FF and supply voltage to satisfy a predefined yield constraint with minimum energy consumption.

In Sect. 5.1, the $V_{\mathrm{DD}_{\min }}$ estimation proposed as Step 1 in the previous section is applied for these circuits. Scalability of the proposed model will be verified because these circuits have different numbers of FFs. In Sect. 5.2, Steps 2-4 of the proposed design strategy are applied. In addition, an insight on the energy optimization by selecting FFs of different transistor sizes using the proposed design strategy is presented in Sect. 5.3.

\subsection{Estimation of $V_{\mathrm{DD}_{\min }}$}

First, we evaluate the proposed estimations of $V_{\mathrm{DD}_{\min }}$. Then the estimations are compared with the results of full circuit simulations. The estimated $V_{\mathrm{DD}_{\text {min }}}$ distributions using the proposed method for differently sized FFs are shown in Fig. 10.

To evaluate the accuracy of these estimations, we also show the histograms of $V_{\mathrm{DD}_{\min }}$ of these circuits obtained through Monte Carlo circuit simulations. "MC" in Fig. 10 shows the $V_{\mathrm{DD}_{\min }}$ distributions where 100 simulation runs are performed for FFs with different transistor $W$ 's; $\times 1, \times 1.5$, and $\times 2$. To obtain accurate $V_{\mathrm{DD}_{\min }}$ through simulation, activation of all paths is needed. Since the initial states of FFs are reset to 0's, we inject test patterns that include a lot of

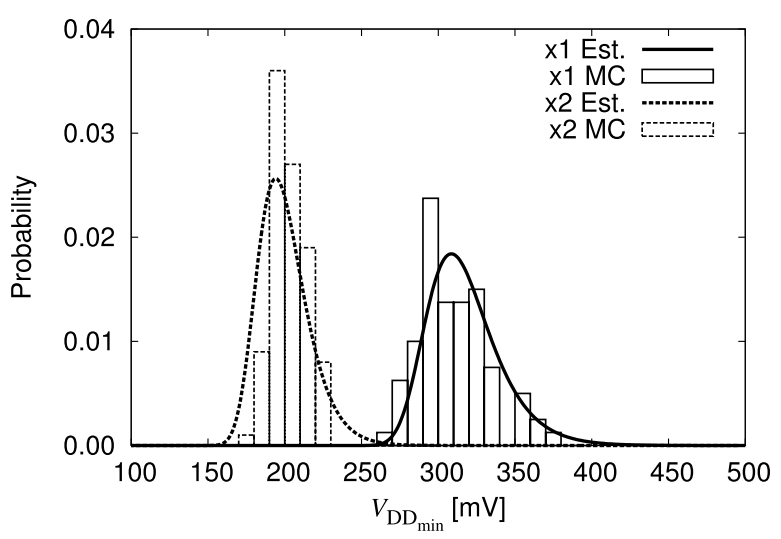

(a) VLC

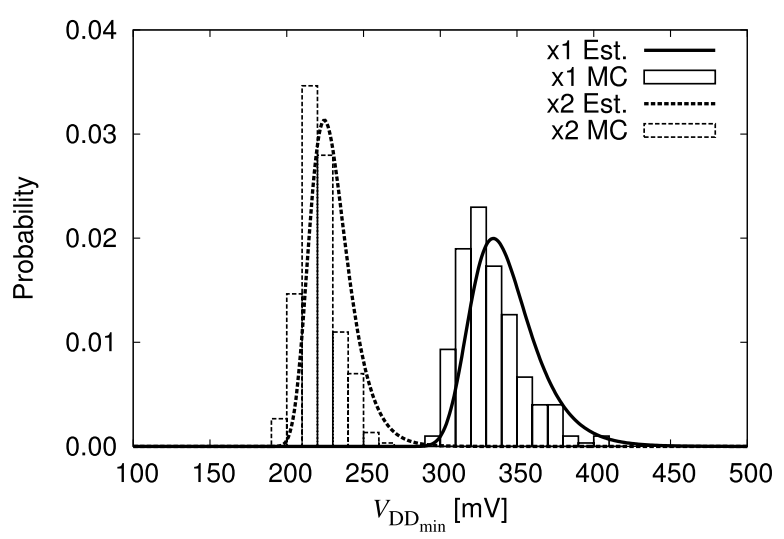

(b) DCT

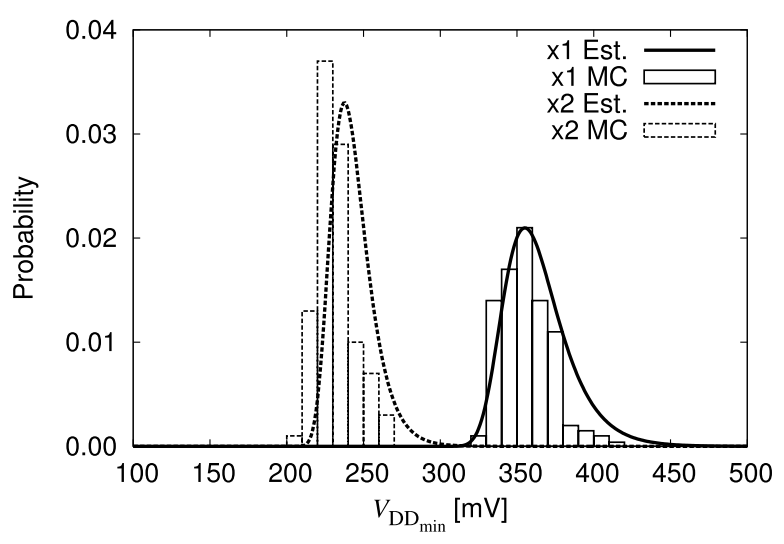

(c) Q

Fig. 10 Estimated $V_{\mathrm{DD}_{\text {min }}}$ distributions using the proposed model (Step 1 of the proposed design strategy), and the histograms obtained by SPICE Monte Carlo simulations. The result for $W: \times 1.5$ is omitted for visibility because it largely overlaps to the results of the other two sized transistors.

1 's in a binary representation (e.g., negative numbers in the 2 's complement, etc.), in the early stage of the test vector to toggle these FFs from 0 to 1 , followed by zero-rich patterns to toggle these FFs again to 0 .

Among these simulations, no timing constraint is assumed, which is the same approach as the former simula- 
Table 2 Required processing time to obtain $V_{\mathrm{DD}_{\min }}$ distribution for each circuit.

\begin{tabular}{c|c|c}
\hline Module & $\begin{array}{c}\text { Proposed method } \\
\text { (Est. in Fig. 10) }\end{array}$ & $\begin{array}{c}\text { Monte Carlo } \\
\text { (MC in Fig. 10) }\end{array}$ \\
\hline \hline VLC & less than 1 sec & 7 days \\
\hline DCT & less than 1 sec & 4 days \\
\hline Q & less than 1 sec & 2 days \\
\hline
\end{tabular}

Table 3 Step 1: Estimation of $V_{\mathrm{DD}_{\text {min }}}$ which guarantees $99 \%$ yield for each circuit.

\begin{tabular}{c||c|c|c}
\hline Tr.size & VLC & DCT & Q \\
\hline \hline$\times 1$ & $398 \mathrm{mV}$ & $418 \mathrm{mV}$ & $432 \mathrm{mV}$ \\
\hline$\times 1.5$ & $308 \mathrm{mV}$ & $323 \mathrm{mV}$ & $335 \mathrm{mV}$ \\
\hline$\times 2$ & $267 \mathrm{mV}$ & $281 \mathrm{mV}$ & $292 \mathrm{mV}$ \\
\hline
\end{tabular}

tions in Fig. 8.

By comparing the distributions in Fig. 10, we can observe that the $V_{\mathrm{DD}_{\min }}$ distributions of all circuits are well approximated by the proposed model. This indicates that $V_{\mathrm{DD}_{\min }}$ for large circuits can be estimated stochastically by tractable circuit simulations only.

Table 2 compares the required time to obtain the $V_{\mathrm{DD}_{\min }}$ distributions by the proposed method and the Monte Carlo simulation. For all three circuit modules, the proposed method requires less than one second to obtain each distribution whereas it takes several days if we resort to the brute force Monte Carlo simulations. Note that, to apply the proposed method, the $V_{\mathrm{DD}_{\text {min }}}$ distribution of an FF shown in Fig. 8 is required. It takes about 5 hours in our case. Once we obtain the $V_{\mathrm{DD}_{\min }}$ distribution of an $\mathrm{FF}$, we can estimate any circuits in a very short time by using Eq. (2).

In this section, an equal yield requirement of $99 \%$ is assumed for all the circuit modules. In this condition, $V_{\mathrm{DD}_{\min }}$ estimations are obtained as shown in Table 3.

\subsection{Evaluation of Energy Consumption and Finding Opti- mal $V_{\mathrm{DD}}$}

Next, we consider the energy consumption of the circuits by applying Steps $2-4$ of the proposed design strategy. Figure 11 shows the total energy required to process one block $(8 \times 8$ pixels $)$ of an image obtained through full circuit simulations. From Fig. 11, it is observed that $V_{\mathrm{ENE}_{\min }}$ of the DCT circuit is relatively low, while that of VLC circuit is relatively high. Since it is known that $V_{\mathrm{ENE}_{\min }}$ is strongly affected by the switching factor $\alpha$ [13], it is inferred that $\alpha$ of the DCT circuit is relatively high, while that of VLC circuit is relatively low. The difference in the ratio of dynamic and static energy changes $V_{\mathrm{ENE}_{\min }}$ of a circuit. For example, the DCT circuit employs pipelined architecture, and thus most of the FFs in the circuit are activated at almost every clock cycle. On the other hand, for the VLC circuit, we applied various input vectors, most of which includes many 0's in higher order components. These are the realistic inputs for a VLC circuit of a JPEG encoder. Circles in this figure indicate $V_{\mathrm{DD}_{\min }}$ values that guarantees a given yield requirement as has been shown in Table 3 .

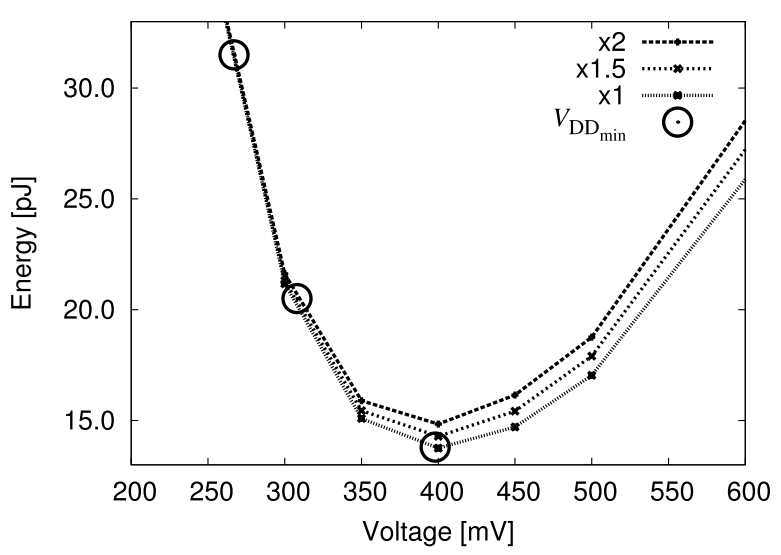

(a) VLC

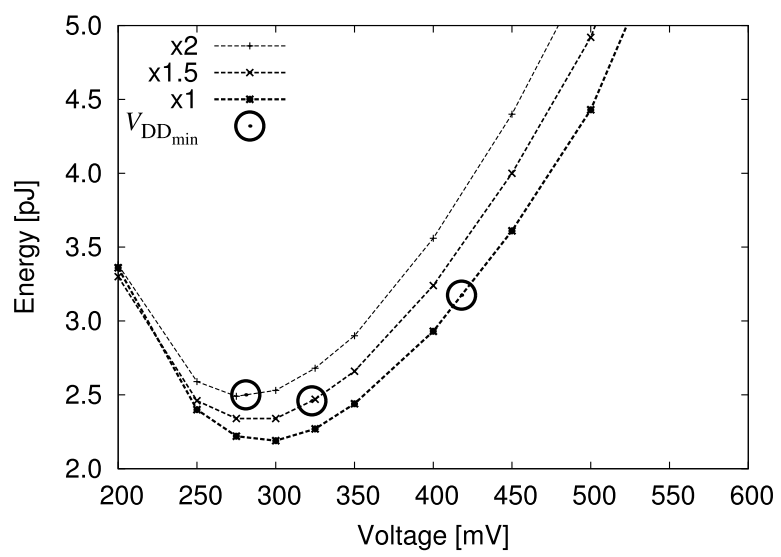

(b) DCT

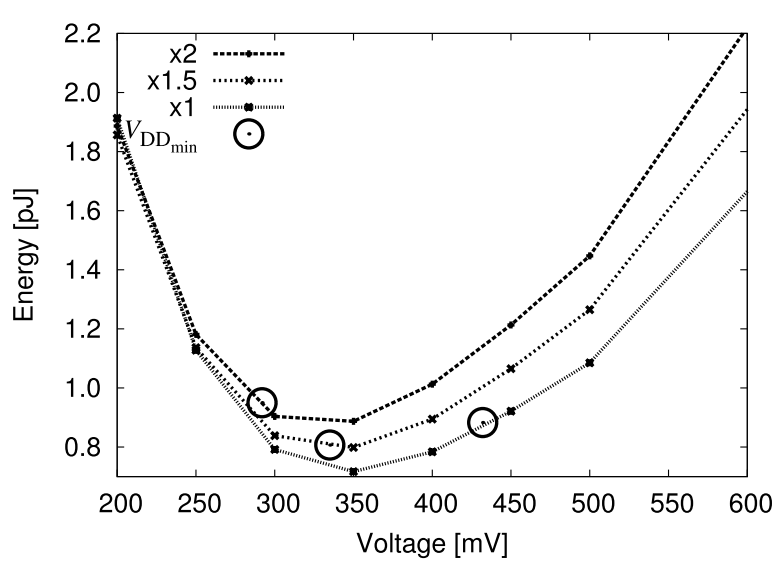

(c) Q

Fig. $11 V_{\mathrm{DD}}$-energy curve with $V_{\mathrm{DD}_{\min }}$ which guarantees a given yield requirement, $99 \%$ (Steps 2 and 3 of the proposed design strategy). The energy is defined as the energy required to process one block ( $8 \times 8$ pixels) of an image.

\section{(1) VLC}

From Table 3, $V_{\mathrm{DD}_{\min }}$ values for the VLC circuit are 398, 308 , and $267 \mathrm{mV}$ using the FFs of $\times 1, \times 1.5$, and $\times 2$, respectively. From Fig. 11(a), the VLC circuit has its own $V_{\mathrm{ENE}_{\min }}$ near $400 \mathrm{mV}$ in all cases. Thus the optimal $V_{\mathrm{DD}}$ is $V_{\mathrm{ENE}_{\min }}$ in all cases, since $V_{\mathrm{ENE}_{\min }}$ is higher than $V_{\mathrm{DD}_{\min }}$. 


\section{(2) DCT}

From Table 3, $V_{\mathrm{DD}_{\min }}$ values for the DCT circuit are 418, 323 , and $281 \mathrm{mV}$ using the FFs of $\times 1, \times 1.5$, and $\times 2$, respectively. From Fig. 11(b), the DCT circuit has its own $V_{\mathrm{ENE}_{\min }}$ near $300 \mathrm{mV}$ in all cases. Thus, the optimal $V_{\mathrm{DD}}$ is $V_{\mathrm{ENE}_{\min }}$ in the case of $\times 2 \mathrm{FF}$, whereas the optimal $V_{\mathrm{DD}}$ is dominated by $V_{\mathrm{DD}_{\text {min }}}$ in the other cases since $V_{\mathrm{DD}_{\text {min }}}$ is higher than $V_{\mathrm{ENE}_{\min }}$.

\section{(3) Quantization}

From Fig. 11(c), the quantization circuit has its own $V_{\mathrm{ENE}_{\min }}$ near $350 \mathrm{mV}$ in all cases. This indicates that in $\times 1.5$ and $\times 2$ cases, we may set the supply voltage at $350 \mathrm{mV}$ for minimizing energy, whereas we need to operate at $432 \mathrm{mV}$ in the $\times 1$ case.

\subsection{Energy Optimization with FF Sizing}

As we have previously seen, $V_{\mathrm{DD}}$ is an important parameter for optimizing energy consumption. From Fig. 11, we can see that the transistor size of the FFs is another important parameter.

As described in Sect. 2, energy consumption takes a minimum value at a supply voltage $V_{\mathrm{ENE}_{\min }}$, and we can operate the circuit at $V_{\mathrm{ENE}_{\min }}$ when $V_{\mathrm{DD}_{\text {min }}}$ is lower than $V_{\mathrm{ENE}_{\min }}$ to meet the given yield constraint. As we have seen in Sect. 3.1, upsizing transistors in the FFs improves $V_{\mathrm{DD}_{\min }}$ at the penalty of increased energy consumption. Upsizing transistors increases (1) dynamic power owing to increased parasitic capacitance, and (2) leakage power owing to decreased resistance. This energy increase should be smaller than the energy savings obtained by lowering the supply voltage. To investigate this trade-off, we review Fig. 11 again.

In the VLC circuit, $V_{\mathrm{DD}_{\text {min }}}$ is lower than $V_{\mathrm{ENE}_{\min }}$ even in the case of the $\times 1$ FF. Thus, there is no benefit of using larger FFs. In the DCT circuit, $V_{\mathrm{DD}_{\min }}$ using the $\times 1 \mathrm{FF}$ is higher than $V_{\mathrm{ENE}_{\min }}$ and transistor sizing contributes to $V_{\mathrm{DD}}$ lowering. However, using the $\times 2 \mathrm{FF}$ is inefficient because it results in excessive energy consumption in total. From Fig. 11(b), we can see that operating at $323 \mathrm{mV}$ using the $\times 1.5 \mathrm{FF}$ is the best choice among the three sizes. It achieves an energy savings of about $20 \%$ compared with the original design of $418 \mathrm{mV}$ using the $\times 1 \mathrm{FF}$. Also, in the quantization circuit, $V_{\mathrm{DD}_{\min }}$ using the $\times 1 \mathrm{FF}$ is higher than $V_{\mathrm{ENE}_{\min }}$. By comparing energies at $432 \mathrm{mV}$ for the $\times 1 \mathrm{FF}$, at $350 \mathrm{mV}$ for the $\times 1.5 \mathrm{FF}$, and at $350 \mathrm{mV}$ for the $\times 2 \mathrm{FF}$, we can determine that using the $\times 1.5 \mathrm{FF}$ at $350 \mathrm{mV}$ is the best choice.

The major drawback of the transistor sizing are the area and performance overheads. As for the area overhead, our $\times 2$ FF cell occupies about $21 \%$ larger area than the $\times 1 \mathrm{FF}$ cell. Assume that all $\times 1 \mathrm{FF}$ cells in the DCT circuit are replaced by the $\times 2$ FF cells, the area occupied by the $\times 1 \mathrm{FF}$ cells $(22.3 \%)$ will increase by $21 \%$, resulting in an overall area overhead of $22.3 \% \times 21 \%=4.68 \%$. Similarly, the overall area overhead for VLC and Q circuits are even smaller than the DCT circuit as $0.40 \%$ and $4.49 \%$, respectively.
As for performance, we performed simulation-based Shmoo plottings for DCT circuit using $\times 2$ FF cells and $\times 1$ FF cells with supply voltage ranging from $1.2 \mathrm{~V}$ to $0.275 \mathrm{~V}$. The observed performance degradation from this experiment is less than $1 \%$ for all voltages.

\section{Conclusion}

In this paper, we investigated a design strategy for subthreshold circuits by simultaneously considering energy minimization and yield maximization.

Regarding yield maximization, minimum supply voltages for three circuits designed by using various transistors of different gate width are efficiently estimated by the proposed method without performing full circuit simulations. It is shown that $V_{\mathrm{DD}_{\min }}$ is dominated by FFs rather than their combinational counterpart, and thus $V_{\mathrm{DD}_{\min }}$ of a large circuit can be stochastically estimated from the distribution of $V_{\mathrm{DD}_{\text {min }}}$ of a very simple circuit, which can be analyzed easily. We also found that the distributions of $V_{\mathrm{DD}_{\min }}$ of the simple circuit are all well approximated by log-normal distributions.

Regarding energy minimization, it is shown that two key voltages are important: the minimum energy consumption voltage and $V_{\mathrm{DD}_{\min }}$. The relative magnitude of these voltages determines the optimal operation voltage in terms of energy consumption. It is also demonstrated that transistor sizing in an FF achieves energy reduction by lowering $V_{\mathrm{DD}_{\text {min }}}$ without significantly increasing energy consumption. Furthermore, gate widths of transistors in FFs are altered as an additional optimization parameter using curves of $V_{\mathrm{DD}_{\text {min }}}$ versus energy.

In the experiments throughout this paper, we did not consider the die-to-die process variation, i.e., we assume that the mean of the threshold voltage distribution equals to the typical threshold voltage. In [8], it is reported that die-to-die variation has a strong impact in $V_{\mathrm{DD}_{\text {min }}}$ especially when the die falls into FS or SF corner. The proposed design strategy is applicable for such process corners if we just perform a simulation similar to Fig. 8 using threshold voltage distribution of the process corner to be considered.

\section{Acknowledgement}

This work is supported by a Grant-in-Aid for Scientific Research (B) 22360143; the JST CREST project; and the VLSI Design and Education Center (VDEC) at the University of Tokyo in collaboration with Synopsys, Inc., and Mentor Graphics, Inc.

\section{References}

[1] J. Kwong, Y.K. Ramadass, N. Verma, and A.P. Chandrakasan, "A $65 \mathrm{~nm}$ sub- $V_{t}$ microcontroller with integrated SRAM and switched capacitor DC-DC converter," IEEE J. Solid-State Circuits, vol.44, no.1, pp.115-126, Jan. 2009.

[2] B.A. Warneke and K.S.J. Pister, "An ultra-low energy microcontroller for smart dust wireless sensor networks," ISSCC Dig. Tech. 
Papers, pp.316-317, Feb. 2004.

[3] G. Chen, M. Fojtik, D. Kim, D. Fick, J. Park, M. Seok, M.T. Chen, Z. Foo, D. Sylvester, and D. Blaauw, "Millimeter-scale nearly perpetual sensor system with stacked battery and solar cells," ISSCC Dig. Tech. Papers, pp.288-289, Feb. 2010.

[4] H. Chen, Y. Jun, Z. Meng, and W. Xiulong, "A 12T subthreshold SRAM bit-cell for medical device application," Proc. International Conference on Cyber-Enabled Distributed Computing and Knowledge Discovery (CyberC), pp.540-543, Oct. 2011.

[5] V. Raghunathan, A. Kansal, J. Hsu, J. Friedman, and M. Srivastava, "Design considerations for solar energy harvesting wireless embedded systems," Proc. International Symposium on Information Processing in Sensor Networks (IPSN), pp.457-462, April 2005.

[6] S. Roundy, E.S. Leland, J. Baker, E. Carleton, E. Reilly, E. Lai, B. Otis, J.M. Rabaey, P.K. Wright, and V. Sundararajan, "Improving power output for vibration-based energy scavengers," IEEE Pervasive Computing, vol.4, no.1, pp.28-36, Jan. 2005.

[7] A. Wang and A.P. Chandrakasan, "A $180 \mathrm{mV}$ FFT processor using subthreshold circuit techniques," ISSCC Dig. Tech. Papers, pp.292529, Feb. 2004.

[8] T. Niiyama, Z. Piao, K. Ishida, M. Murakata, M. Takamiya, and T. Sakurai, "Increasing minimum operating voltage $\left(\mathrm{V}_{\mathrm{DDmin}}\right)$ with number of CMOS logic gates and experimental verification with up to 1mega-stage ring oscillators," Proc. International Symposium on Low Power Electronics and Design (ISLPED), pp.117-122, Aug. 2008

[9] B.H. Calhoun and A.P. Chandrakasan, "Ultra-dynamic voltage scaling (UDVS) using sub-threshold operation and local voltage dithering," IEEE J. Solid-State Circuits, vol.41, no.1, pp.238-245, Jan. 2006.

[10] A.P. Chandrakasan, S. Sheng, and R.W. Brodersen, "Low-power CMOS digital design," IEEE J. Solid-State Circuits, vol.27, no.4, pp.473-484, April 1992.

[11] B.H. Calhoun, J.F. Ryan, S. Khanna, M. Putic, and J. Lach, "Flexible circuits and architectures for ultralow power," Proc. IEEE, vol.98, no.2, pp.267-282, Feb. 2010.

[12] M.J.M. Pelgrom, A.C.J. Duinmaijer, and A.P.G. Welbers, "Matching properties of MOS transistors," IEEE J. Solid-State Circuits, vol.24, no.5, pp.1433-1439, Oct. 1989.

[13] B. Zhai, D. Blaauw, D. Sylvester, and K. Flautner, "Theoretical and practical limits of dynamic voltage scaling," Proc. DAC, pp.868 873 , July 2004

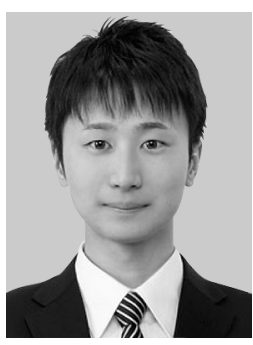

Junya Kawashima received his B.E. degree in Electrical and Electronic Engineering from Kyoto University in 2011. Presently, he is a master course student at Department of Communications and Computer Engineering, Kyoto University.

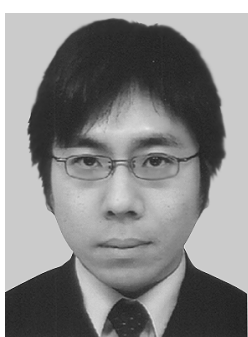

Hiroshi Tsutsui received his B.E. degree in Electrical and Electronic Engineering and his master and Ph.D. degrees in Communications and Computer Engineering from Kyoto University in 2000, 2002, and 2005, respectively. $\mathrm{He}$ is currently an assistant professor in the Department of Communications and Computer Engineering, Kyoto University. His research interests include circuits and systems for image processing and VLSI design methodology. He is a member of IEEE, ACM, IPSJ, IEEJ, and IIEEJ.

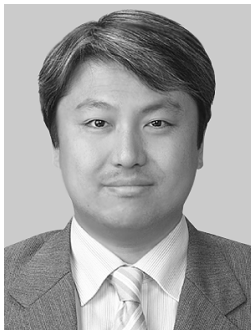

Hiroyuki Ochi received the B.E., M.E., and Ph.D. degrees in Engineering from Kyoto University in 1989, 1991, and 1994, respectively. In 1994, he joined Department of Computer Engineering, Hiroshima City University as an associate professor. Since 2004, he has been an associate professor of Department of Communications and Computer Engineering, Kyoto University. His research interests include lowpower/reliability-aware VLSI design and reconfigurable architectures. He is a member of IPSJ,

IEEE, and ACM.

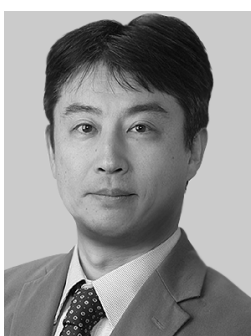

Takashi Sato received B.E. and M.E. degrees from Waseda University, Tokyo, Japan, and a Ph.D. degree from Kyoto University, Kyoto, Japan. He was with Hitachi, Ltd., Tokyo, Japan, from 1991 to 2003, with Renesas Technology Corp., Tokyo, Japan, from 2003 to 2006, and with the Tokyo Institute of Technology, Yokohama, Japan. In 2009, he joined the Graduate School of Informatics, Kyoto University, Kyoto, Japan, where he is currently a professor. He was a visiting industrial fellow at the University of California, Berkeley, from 1998 to 1999. His research interests include CAD for nanometer-scale LSI design, fabrication-aware design methodology, and performance optimization for variation tolerance. Dr. Sato is a member of the IEEE. He received the Beatrice Winner Award at ISSCC 2000 and the Best Paper Award at ISQED 2003. 